\title{
The LHC Signatures of the Extended Gauge Structures in Supersymmetry
}

\author{
Ahmed Ali \\ Deutsches Elektronen - Synchrotron DESY, Notkestr. 85, D-22603 Hamburg, Germany \\ E-mail: ahmed.ali@desy.de
}

\section{Durmuş A. Demir*}

Department of Physics, Izmir Institute of Technology, IZTECH, TR35430 Izmir, Turkey

E-mail: demir@physics.iztech.edu.tr

\section{Mariana Frank}

Department of Physics, Concordia University, 7141 Sherbrooke St. West, Montreal, Quebec, CANADA H4B IR6

E-mail: mfrankealcor.concordia.ca

\section{Ismail Turan}

Department of Physics, Concordia University, 7141 Sherbrooke St. West, Montreal, Quebec, CANADA H4B IR6

E-mail: ituran@physics.concordia.ca

\begin{abstract}
The extensions of the MSSM, driving mainly from the need to solve the $\mu$ problem, involve novel matter species and gauge groups. These extended MSSM models can be searched for at the LHC via gauge bosons or their fermionic partners. Traditionally, the focus has been on the gauge $\left(Z^{\prime}\right)$ bosons. However, their fermionic partners offer an alternative way. In this talk, we thus discuss a $U(1)^{\prime}$ gauge extension of the MSSM, whose LHC signatures are analyzed via productions and decays of the scalar quarks and gluinos, which will be produced copiously at the LHC. We perform a detailed simulation study of the events of the type $p p \rightarrow n$ leptons $+m$ jets $+\mathbb{E}_{T}$, and compare the results with those of the MSSM. We conclude that searches for $U(1)^{\prime}$ can proceed via gauge fermions as well. $U(1)^{\prime}$ searches based on events induced by squark/gluino decays are complementary to the direct $Z^{\prime}$ searches at the LHC.
\end{abstract}

European Physical Society Europhysics Conference on High Energy Physics, EPS-HEP 2009, July 16 - 222009

Krakow, Poland

\footnotetext{
${ }^{*}$ Speaker.
} 


\section{Introduction}

The search for the non-SM gauge interactions is of particular importance since non-SM gauge forces at the weak scale can give important hints about the symmetries of Nature at short distances.

The search strategies for, and the signatures of, the extra gauge interactions depend crucially on the structure of the model of 'new physics'. Supersymmetric theories offer a viable framework for elucidating these observations, as in these theories the entire particle spectrum is paired to have the boson-fermion symmetry, and thus, quadratic divergences that destabilize the scalar field sector are naturally avoided. In particular, gauge bosons themselves are paired with the corresponding gauge fermions, and this feature guarantees that any scattering process involving the gauge bosons possesses a partner process proceeding with the gauge fermions (along with the exchange of fermions and scalar fermions). This implies that the search for extended gauge structures can be performed via both gauge bosons and gauge fermions, and the correlations between the two can reveal the underlying supersymmetric structure.

In this talk, we present simulation results [1] for the LHC signatures of an extended gauge structure within a supersymmetric framework by specializing to a simple $G_{G M} \otimes U(1)^{\prime}$ gauge extension of the MSSM. We hereon call this extended supersymmetric gauge theory simply as $U(1)^{\prime}$ model.

\section{The $U(1)^{\prime}$ Model and Its LHC Signatures}

There are various reasons for extending the MSSM by an additional $U(1)$ group. From the point of view of high energies, an extra $U(1)$ symmetry broken at the $\mathrm{TeV}$ scale frequently arises in grand unified theories and strings. Seen from the low energy point of view, introduction of an extra $U(1)$ is motivated by the need to solve the $\mu$ problem of the MSSM (see $[1,2]$ and references therein). The $\mu \widehat{H}_{u} \cdot \widehat{H}_{d}$ bilinear in the MSSM is replaced by $h_{s} \widehat{S} \widehat{H}_{u} \cdot \widehat{H}_{d}$ where the $U(1)^{\prime}$ charges of $\widehat{S}$ and those of $\widehat{H}_{u, d}$ sum up to zero. The $\widehat{S}$, a chiral field not found in the MSSM, induces the $\mu$ parameter as $\mu_{e f f}=h_{s}\langle S\rangle$, with obvious $U(1)^{\prime}$ protection. The $U(1)^{\prime}$ gaugino is another field that adds to the MSSM spectrum. The hypercharge and $U(1)^{\prime}$ kinetic terms mix, and hence $\mathscr{L}_{\text {gauge }} \ni(1 / 32)\left(\widehat{W}_{Y} \widehat{W}_{Y}+\widehat{W}_{Y^{\prime}} \widehat{W}_{Y^{\prime}}+2 \sin \chi \widehat{W}_{Y} \widehat{W}_{Y^{\prime}}\right)_{F}$ where $\chi$ is the mixing angle. Then, $\mathscr{L}_{\text {gaugino }} \ni-(1 / 2) M_{\widetilde{B}} \widetilde{B} \widetilde{B}-(1 / 2) M_{\widetilde{Z}} \widetilde{Z}^{\prime} \widetilde{Z}^{\prime}-M_{\widetilde{Y} \widetilde{Z^{\prime}}} \widetilde{B} \widetilde{Z}^{\prime}$. Here, original fields $\left(\widetilde{Y}, \widetilde{Y}^{\prime}\right)$ are rotated to kill the kinetic mixing so that new gauginos $\left(\widetilde{B}, \widetilde{Z^{\prime}}\right)$ obtain the masses $M_{\widetilde{B}}, M_{\widetilde{Z} !}$, and mass mixing $M_{\widetilde{B} \widetilde{Z} !}$

$$
M_{\widetilde{B}}=M_{\widetilde{Y}}, \cos ^{2} \chi M_{\widetilde{Z^{\prime}}}=M_{\widetilde{Y}^{\prime}}-2 \sin \chi M_{\widetilde{Y} \widetilde{Y}^{\prime}}+M_{\widetilde{Y}} \sin ^{2} \chi, \cos \chi M_{\widetilde{B} \widetilde{Z}^{\prime}}=M_{\widetilde{Y} \widetilde{Y}^{\prime}}-M_{\widetilde{Y}} \sin \chi .
$$

The collider search for $U(1)^{\prime}$ is based on signals of $\left(Z_{\mu}^{\prime}, \widetilde{Z}^{\prime}\right)$ and $(S, \widetilde{S})$. Search strategies for bosonic states have been discussed in literature [3]. Our concern is on the fermionic states. The squarks/gluinos will be produced copiously at the LHC, and they provide a perfect place to search for effects of $\widetilde{Z} \widetilde{Z}^{\prime}$. The reason is that decay channels of squarks change with the branching brought about by the $\widetilde{Z}^{\prime}$ coupling. The channels are depicted in Table 1 . In simulation studies, we use $\mathscr{R}_{Y^{\prime}} \equiv \frac{M_{\tilde{Y}}{ }^{\prime}}{M_{\tilde{Y}}}$ and $\mathscr{R}_{Y Y^{\prime}} \equiv \frac{M_{\tilde{Y} \tilde{Y}^{\prime}}}{M_{\tilde{Y}}}$ as free parameters. We compute the cross sections and branching ratios, and generate parton-level events by using CalcHEP $v .2 .5$. We modified the package to incorporate the features pertaining to the $U(1)^{\prime}$ model with the help of LanHEP Package. Hadronization (including initial and final state radiations) and restrictions imposed by various cuts have been 


\begin{tabular}{ccc}
\hline \hline Sfermion & MSSM & \multicolumn{1}{c}{$U(1)^{\prime}$ Model } \\
\hline \hline$\widetilde{f}_{R}$ & $\widetilde{f}_{R} \rightarrow f_{R} \widetilde{B}$ & $\widetilde{f}_{R} \rightarrow f_{R} \widetilde{B} \oplus \widetilde{f}_{R} \rightarrow f_{R} \widetilde{Z}^{\prime}$ \\
\hline \multirow{2}{*}{$\widetilde{f}_{L}$} & $\widetilde{f}_{L} \rightarrow f_{L} \widetilde{B}$ & $\widetilde{f}_{L} \rightarrow f_{L} \widetilde{B}$ \\
& $\widetilde{f}_{L} \rightarrow f_{L} \widetilde{W}^{3}$ & $\widetilde{f}_{L} \rightarrow f_{L} \widetilde{W}^{3} \oplus \widetilde{f}_{L} \rightarrow f_{L} \widetilde{Z}^{\prime}$ \\
\hline \hline
\end{tabular}

Table 1: The decay channels of the scalar fermions $\widetilde{f}$ into gauginos in the MSSM and the U(1)' model.
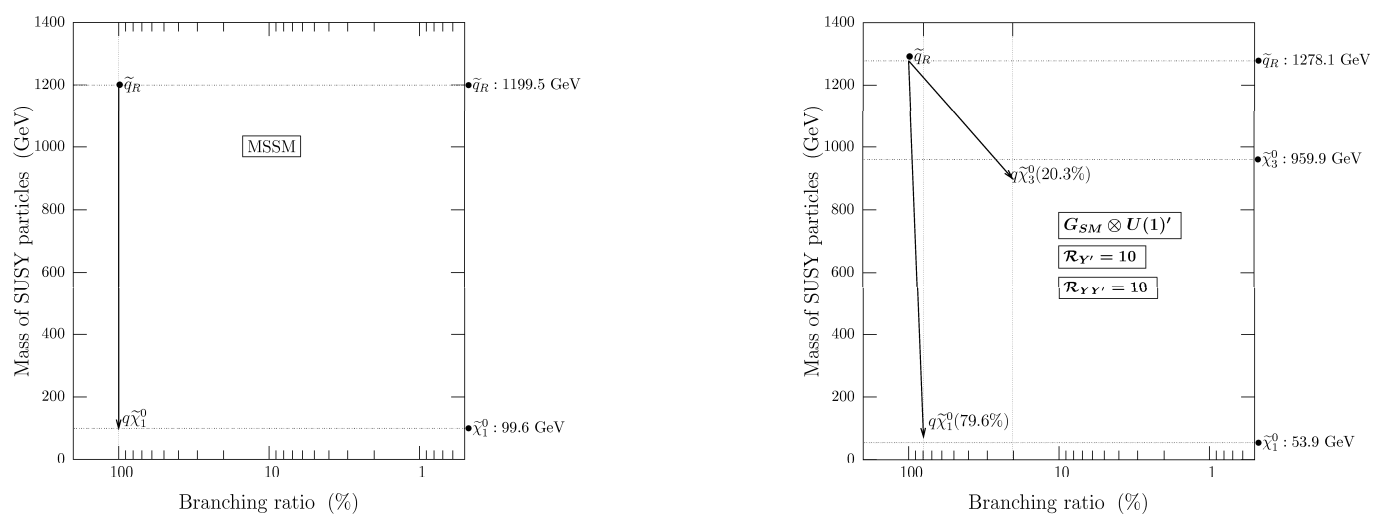

Figure 1: The branching ratio (\%) of a right-handed squark (in 1st or 2nd family) into a quark and a neutralino (left panel: MSSM, right panel: $U(1)^{\prime}$ model).

achieved with PYTHIA by using the CalCHEP-PYTHIA interface. The parton distributions in the proton have been parametrized by using CTEQ6L of LHAPDF (see [1] and references therein).

The parameter values used are: $m_{\widetilde{q}_{L}}=m_{\widetilde{q}_{R}}=1200 \mathrm{GeV}, m_{\widetilde{e}_{L}}=350 \mathrm{GeV}, m_{\widetilde{e}_{R}}=200 \mathrm{GeV}, M_{\widetilde{Y}}=$ $100 \mathrm{GeV}, M_{\widetilde{W}}=400 \mathrm{GeV}, M_{\widetilde{g}}=1300 \mathrm{GeV}$ and $h_{s}=0.6, \mu_{\text {eff }}=1400 \mathrm{GeV}, \tan \beta=10, \sin \chi=$ $5 \times 10^{-3}$. The $U(1)^{\prime}$ invariance corresponds to $U(1)_{\eta}$ from $E_{6}$, and $M_{Z^{\prime}}=933 \mathrm{GeV}$.

Depicted in Fig. 1 is the branching ratio of $\widetilde{q}_{R} \rightarrow q \chi_{i}^{0}(i=1, \ldots, 6)$. In the MSSM, shown in left panel, $\widetilde{q}_{R}$ decays into $\chi_{1}$ (dominated by the bino $\widetilde{B}$ ). In $U(1)^{\prime}$ model, however, the situation is different in that $\widetilde{Z}^{\prime}$ exchange opens up new decay channels to other neutralinos such as $\chi_{3}^{0}$. The new branching is not small; it is $\sim 20 \%$.

Depicted in Fig. 2 are missing energy and dilepton invariant mass distributions. Shown in upper two panels are the purely hadronic (left panel) and single-lepton (right panel) events. Each panel is plotted for the MSSM and two distinct choices for $\left(\mathscr{R}_{Y^{\prime}}, \mathscr{R}_{Y Y^{\prime}}\right)$. The two panels manifestly confirm opening of $\widetilde{Z}^{\prime}$-induced channel shown in Table 1 as well as Fig. 1. The left-panel shows that the number of purely hadronic events decreases in $U(1)^{\prime}$ model. The right-panel, on the other hand, indicates that leptonic events increase in $U(1)^{\prime}$ model. Both features are directly expected from Fig. 1.

The lower two panels of Fig. 2 show dileptonic invariant mass distributions for dilepton events (left panel) and trilepton events (right panel). The difference between MSSM and $U(1)^{\prime}$ distributions are manifest. The distributions in $U(1)^{\prime}$ case extend to larger invariant masses compared to the MSSM. The dileptons are more massive in $U(1)^{\prime}$ than in MSSM. The comparative analysis here 

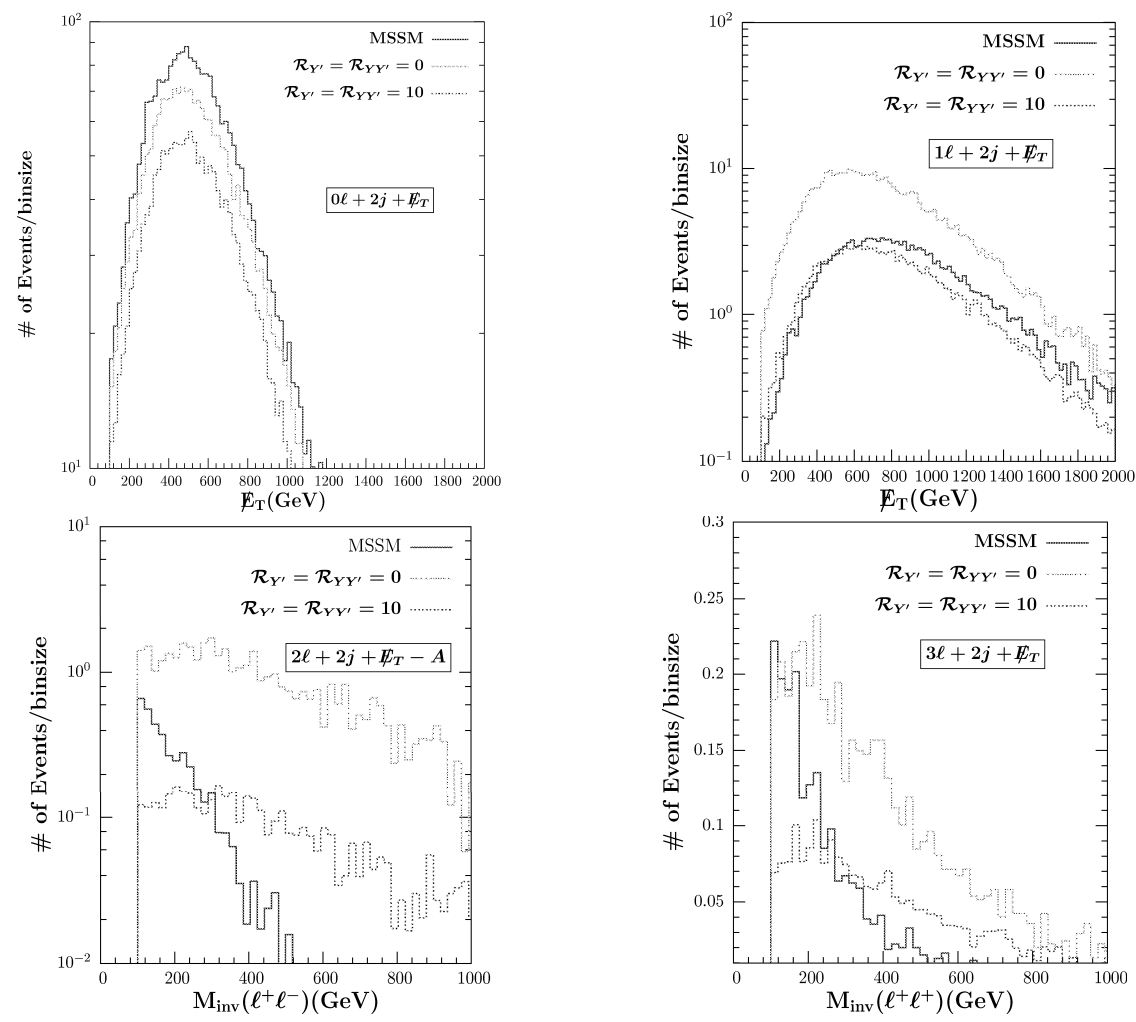

Figure 2: The number of events per binsize for missing energy distribution (upper two panels) and for dilepton invariant mass distribution (lower two panels). The insets give details.

extends to several other observables, and MSSM and $U(1)^{\prime}$ exhibit distinguishable features [1].

\section{Conclusion}

In this talk, we have discussed an alternative way for searching for new gauge structures in supersymmetry, and exemplified it by specializing to a $U(1)^{\prime}$ extension of the MSSM. The results show that extended gauge structures can be distinguished from the MSSM, and they can be established at the LHC via cascade decays of the squarks/gluinos which will be produced copiously at the LHC.

\section{References}

[1] A. Ali, D. A. Demir, M. Frank and I. Turan, Phys. Rev. D 79 (2009) 095001 [arXiv:0902.3826 [hep-ph]].

[2] M. Cvetic, D. A. Demir, J. R. Espinosa, L. L. Everett and P. Langacker, Phys. Rev. D 56 (1997) 2861 [Erratum-ibid. D 58 (1998) 119905] [arXiv:hep-ph/9703317].

[3] P. Langacker, arXiv:0801.1345 [hep-ph]. 